\title{
丙二酸二甲酯均相催化加氢合成 3-羟基丙酸甲酯的研究
}

\author{
方霄龙 ${ }^{*, a, b}$ 段 宁 ${ }^{a}$ 章 敏 ${ }^{a}$ 张春燕 ${ }^{b}$ 刘 㝵 ${ }^{b}$ 朱红平*,b \\ $\left({ }^{a}\right.$ 池州学院化学与材料工程学院 池州 247000) \\ ( ${ }^{b}$ 厦门大学化学化工学院 固体表面物理化学国家重点实验室 醇梄酯清洁生产国家工程实验室 厦门 361005)
}

\begin{abstract}
摘要 以乙酰丙酮钓和膦胺配体作为催化剂体系, 用于催化丙二酸二甲酯加氢制 3-羟基丙酸甲酯. 围绕催化反应性能, 系统探讨了配体结构和用量、温度、时间以及溶剂等对丙二酸二甲酯转化率和 3-差基丙酸甲酯产率的影响. 研究发现, 乙酰丙酮钓和 $o$-二苯基膦苯胺构成的催化剂体系在合适反应条件下可以有效催化丙二酸二甲酯选择性加氢制 3 -羟基丙 酸甲酯. 在优化的反应条件下, 这一催化体系也可以催化多种不同结构酯类分子加氢制醇.
\end{abstract}

关键词 乙酰丙酮钉; $o$-二苯基膦苯胺; 丙二酸二甲酯; 3 -羊至基丙酸甲酯

\section{Homogeneous Catalytic Hydrogenation of Dimethyl Malonate into Methyl 3-Hydroxypropanoate}

\author{
Fang, Xiaolong *,a,b \\ Duan, Ning ${ }^{a}$ \\ Zhang, $\operatorname{Min}^{a}$ \\ Zhang, Chunyan ${ }^{b}$ \\ Liu, Rui ${ }^{b}$ \\ Zhu, Hongping*,b \\ $\left({ }^{a}\right.$ College of Chemistry and Materials Engineering, Chizhou University, Chizhou 247000) \\ ( ${ }^{b}$ State Key Laboratory of Physical Chemistry of Solid Surfaces, National Engineering Laboratory for Green Chemical Pro- \\ ductions of Alcohols-Ethers-Esters, College of Chemistry and Chemical Engineering, Xiamen University, Xiamen 361005)
}

\begin{abstract}
Ruthenium acetylacetonate and aminophosphine ligand were selected as the catalyst system and applied to the catalytic hydrogenation of dimethyl malonate into methyl 3-hydroxypropanoate. With the focus on the catalytic efficiency, the important factors with significant influences on the dimethyl malonate conversion and methyl 3-hydroxypropanoate selectivity were well discussed, including the structure and dosage of the ligand, temperature, reaction time, solvent, and so on. The results revealed that catalyst system of ruthenium acetylacetonate and $o$-(diphenylphosphino)aniline ligand could obtain significant catalytic results. Under the optimal reaction conditions, this catalyst system can also be applied to catalytic hydrogenation of some other esters with high efficiency.
\end{abstract}

Keywords ruthenium acetylacetonate; $o$-(diphenylphosphino)aniline; dimethyl malonate; methyl 3-hydroxypropanoate

1,3-丙二醇(1,3-propanediol, 1,3-PDO)是合成聚酯的 重要原料 ${ }^{[1]}$, 近年来 $1,3-\mathrm{PDO}$ 的制备研究备受关注. 目 前, 1,3-PDO 主要通过石化路线获得, 如丙烯醛水合氢 化法 ${ }^{[2]}$ 和环氧乙烷羰基化法 ${ }^{[3]}$. 此外微生物发酵法 ${ }^{[4]} 、 甘$ 油氢解法 ${ }^{[5]}$ 等也是很多课题组研究开发的路线. 随着近 年来煤基合成气路线经草酸二酯、进而加氢制备乙醇酸 酯以及乙二醇研究的开展 ${ }^{[6]}$, 可比拟的丙二酸酯加氢制 备 1,3-PDO 的技术路线研究引起化学家们的特别关 注 $^{[7]}$. 丙二酸二甲酯(dimetyl malonate, DMM)在催化加 氢过程中主要发生的反应如图 1 所示, DMM 首先部分
加氢得到中间产物 3-羞基丙酸甲酯(3-hydroxypropanoic acid methyl ester, 3-HPM), 进一步加氢得到最终产物 1,3-PDO. 但是, DMM 容易发生脱羧反应生成乙酸甲酯, 同时 3-HPM 的 $\beta$ 位羟基稳定性较低，易在酸性位点脱水 转化生成丙烯酸甲酯、丙酸甲酯(methyl propionate, MP) 等副产物. 因此, DMM 高选择性催化加氢制备 3-HPM 是这一反应路线的核心步骤.

目前, 催化丙二酸酯加氢多使用负载型多相催化 剂, 反应条件苛刻 ${ }^{[8]}$. 众所周知, 均相催化剂可在较为 温和条件下高选择性地获取目标产物, 相比于多相催化

\footnotetext{
* Corresponding authors. E-mail: xlfang@stu.xmu.edu.cn; hpzhu@xmu.edu.cn

Received December 10, 2018; revised January 10, 2019; published online January 31, 2019.

Project supported by the National Natural Science Foundation for Young Scientists of China (No. 21802010), the Natural Science Foundation of Anhui Province (No. 1808085QB48), and the Starting Grants for Young Teachers of Chizhou University (No. 2018YJRC001).

国家自然科学基金青年基金(No. 21802010)、安徽省自然科学基金(No. 1808085QB48)及池州学院青年教师科研启动基金(No. 2018YJRC001)资助项目.
} 


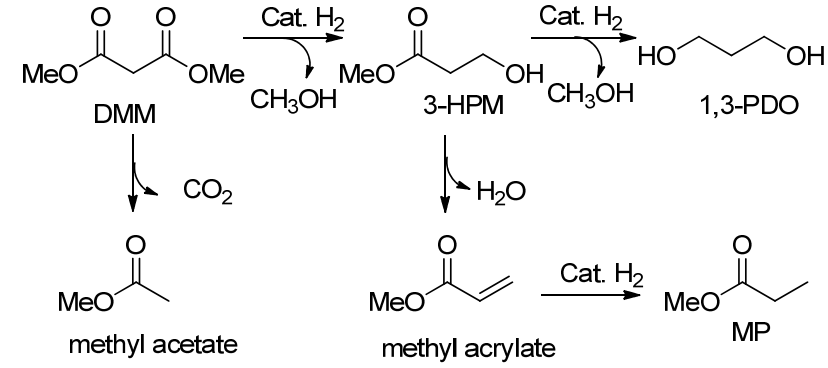

图 1 催化丙二酸二甲酯加氢反应示意图

Figure 1 Reaction process for the catalytic hydrogenation of dimethyl malonate

剂有明显优势 ${ }^{[9]}$. 近年来, 国内 $\mathrm{Zhou}^{[10]}$ 、Ding ${ }^{[1]}$ 、 Zhang $^{[12]}$ 等课题组在均相催化加氢领域开展了众多富有 意义的工作, 取得了显著的研究成果. 最近, 在草酸二 甲酯均相催化加氢制乙醇酸甲酯及乙二醇方面进行了 系列研究 ${ }^{[13]}$, 在此基础上, 进一步将研究工作扩展到丙 二酸酯的催化加氢. 本文中, 采用系列膦胺配体 $\mathbf{L 1} \sim$ $\mathbf{L 8}$ (图 2), 分别与乙酰丙酮钓 $\left[\mathrm{Ru}(\mathrm{acac})_{3}\right]$ 组成催化剂体 系, 考察了催化丙二酸酯加氢的反应性能. 同时选择 L1-Ru(acac) 3 体系, 研究了对其它酯类分子 $\mathbf{E 2} \sim \mathbf{E}$ 7(图 2)的催化加氢性能.<smiles>Nc1ccccc1Pc1ccccc1</smiles>

L1<smiles>[13CH3]/N=C(\c1ccc[nH]1)c1ccccc1P</smiles>

L2<smiles>C(=N\c1ccccc1Pc1ccccc1)\c1ccccc1</smiles>
L3<smiles></smiles>
L4<smiles>NCCc1ccccc1</smiles>
L6<smiles>NCc1ccccc1-c1ccccc1</smiles>

L7<smiles>NCCCc1ccccc1</smiles>

L8<smiles>COC(=O)CC(=O)OC</smiles>

E1<smiles>COC(=O)CCO</smiles>

E2<smiles>CCOC(=O)C(C)OC</smiles><smiles>[R5]CCC(CC)OC(=O)C(=O)OCC</smiles>

图 2 文中用到的配体和酯类化合物

Figure 2 Ligands and esters used in this study

\section{1 结果与讨论}

\section{1 催化剂的篮选}

自 1995 年 Noyori 等 ${ }^{[14]}$ 报道发现膦配体和胺配体构 成的 $\mathrm{Ru}(\mathrm{II})$ 催化剂体系可有效催化醛、酮等羰基衍生物 的加氢反应以来, 这一催化体系的合成及应用研究取得 了显著的进展 ${ }^{[9]}$. 近年来, 我们课题组合成了多种膦胺 配体构成的 $\mathrm{Ru}(\mathrm{II})$ 化合物, 并成功应用于草酸酯、脂肪 酸酯等多种酯类分子的加氢反应研究中 ${ }^{[13 a, 13 b]}$, 但将此
类催化剂应用于 DMM 催化加氢制 3-HPM 或 1,3-PDO 时结果不甚理想. 1997 年, Elsevier 等 ${ }^{[15]}$ 报道采用 $\mathrm{Ru}(\mathrm{acac})_{3}$ 作为金属前驱体, 与膦配体构成催化剂体系. 在甲醇溶液中, 这一体系可有效催化草酸二甲酯加氢制 乙二醇. 紧接着, 该课题组 ${ }^{[16]}$ 研究发现这一催化剂体系 也可以催化芳香酸酯的加氢反应. 2010 年以来, Leitner 等 ${ }^{[17]}$ 进一步将这一催化剂体系应用于生物质酸、 $\mathrm{CO}_{2}$ 等 加氢难度较高底物分子的加氢反应研究中, 均取得不错 的催化反应效果. 基于上述研究进展, 我们尝试采用 $\mathrm{Ru}(\mathrm{acac})_{3}$ 和膦胺配体构成催化体系, 应用于 DMM 均相 催化加氢.

类比文献反应条件 ${ }^{[15]}$, 首先以甲醇为溶剂，在 393 $\mathrm{K}$ 、初始 $\mathrm{H}_{2}$ 压力 $7 \mathrm{MPa}$ 、反应时间 $16 \mathrm{~h}$ 以及 $\mathrm{Ru}(\mathrm{acac})_{3}$ 和配体用量分别为 $1 \mathrm{~mol} \%$ 、2 $\mathrm{mol} \%$ 条件下考察了配体 对体系催化加氢性能的影响. 如表 1 所示, L1-Ru(acac) 3 在反应条件下取得了 $55 \%$ 的 DMM 转化率和 $45 \%$ 的 3-HPM 收率(Entry 1, 表 1). 有趣的是, 将 $\mathbf{L 1}$ 结构中的 胺氢替换为甲基所得的配体 $\mathbf{L 2}$ 所构成的催化体系没有 催化活性(Entry 2, 表 1), 类似的实验现象文献已有报 道 ${ }^{[11,18]}$. 衍生于 $\mathbf{L 1}$ 配体的 $\mathbf{L 3} \sim \mathbf{L 5}$ 分别组成的催化体系 表现出不同的催化活性(Entries 3 6, 表 1). 具体来说, L3-Ru(acac) 3 催化活性低于 L1-Ru(acac) 3 (Entry 3，表 1)， $\mathbf{L} 5-\mathrm{Ru}(\mathrm{acac})_{3}$ 催化活性与 $\mathbf{L 1}-\mathrm{Ru}(\mathrm{acac})_{3}$ 基本相当(Entry 5 , 表 1), 而 L4-Ru(acac) 3 基本没有催化活性(Entry 4, 表 1). 由于 $\mathbf{L} 4$ 配体与 $\mathrm{Ru}$ 中心可以采用 N,N,P-三齿螯合配位, 过多配位基堵塞活性中心，因此 $\mathbf{L 4}-\mathrm{Ru}(\mathrm{acac})_{3}$ 催化活性 低. 相比之下，配体 $\mathbf{L 5}$ 与 $\mathbf{L 1}$ 相似, 采用双齿与金属中 心螯合，所构成体系催化活性相当. L3-Ru(acac) $)_{3}$ 催化活 性低于 $\mathbf{L 1}-\mathrm{Ru}(\mathrm{acac})_{3}$ 和 $\mathbf{L 5}-\mathrm{Ru}(\mathrm{acac})_{3}$ 的原因, 可能是由 于 $\mathbf{L 3}$ 结构中氮原子所连基团的空间位阻较大所致. 在 相同反应条件下, 较为柔软的双齿膦胺配体 $\mathbf{L 6} \sim \mathbf{L 8}$ 组 成的催化体系性能均较差(Entries 7 9, 表 1).

表 1 配体对催化 DMM 加氢反应的影响 ${ }^{a}$

Table 1 Influence of ligands on the catalytic hydrogenation of DMM

\begin{tabular}{ccccccc}
\hline \multirow{2}{*}{ Entry } & \multirow{2}{*}{ Ligand } & \multirow{2}{*}{ Conv./\% } & \multicolumn{5}{c}{ Yield/\% } \\
\cline { 4 - 7 } & & & 3-HPM & 1,3-PDO & MP & Others $^{b}$ \\
\hline 1 & L1 & 55 & 45 & 1 & 3 & 6 \\
2 & L2 & 0 & - & - & - & - \\
3 & L3 & 33 & 25 & 0 & 2 & 6 \\
4 & L4 & 6 & 2 & 0 & 0 & 4 \\
5 & L5 & 55 & 43 & 0 & 8 & 4 \\
6 & L6 & 16 & 6 & 0 & 2 & 8 \\
7 & L7 & 19 & 10 & 0 & 3 & 6 \\
8 & L8 & 9 & 0 & 0 & 1 & 8 \\
\hline
\end{tabular}

${ }^{a}$ Reaction conditions: DMM (7.5 mmol), Ru(acac) $)_{3}(1 \mathrm{~mol} \%)$, ligand (2 mol\%), methanol $(10 \mathrm{~mL})$, temp. $=393 \mathrm{~K}, p\left(\mathrm{H}_{2}\right)=7 \mathrm{MPa}$, time $=16 \mathrm{~h}$.

${ }^{b}$ Mainly containing methyl acetate and methyl acrylate. 
选择最优配体 L1, 进一步考察了配体用量对体系 催化性能的影响, 结果如表 2 所示. 可以看出, 当 $n(\mathbf{L 1}) / n\left(\mathrm{Ru}(\mathrm{acac})_{3}\right)$ 比值为 2 时体系取得了最优的催化反 应结果(Entries 2 4, 表 2), 低于或高于这一比值均不 利于 DMM 酯基加氢反应(Entries $2 \& 4$, 表 2). 值得注 意的是, 当没有使用配体时, 反应体系无催化活性 (Entry 1, 表 2). 综合以上结果, 在下文我们选用物质的 量比为 $2: 1$ 的 $\mathbf{L 1}$ 与 $\mathrm{Ru}(\mathrm{acac})_{3}$ 作为最优的催化体系开 展进一步研究.

表 $2 \mathrm{L1}$ 用量对催化 DMM 加氢反应的影响 ${ }^{a}$

Table 1 The influence of the dosage of $\mathbf{L 1}$ on the catalytic hydrogenation of DMM

\begin{tabular}{cccccccc}
\hline \multirow{2}{*}{ Entry } & \multirow{2}{*}{$n(\mathbf{L 1}) / n(\mathrm{Ru})$} & \multirow{2}{*}{ Conv./\% } & \multicolumn{5}{c}{ Yield $/ \%$} \\
\cline { 4 - 7 } & & & $3-\mathrm{HPM}$ & 1,3 -PDO & MP & Others $^{b}$ \\
\hline 1 & 0 & 0 & 0 & 0 & 0 & 0 \\
2 & 1 & 14 & 10 & 0 & 1 & 3 \\
3 & 2 & 55 & 45 & 1 & 3 & 6 \\
4 & 3 & 3 & 1 & 0 & 0 & 2 \\
\hline
\end{tabular}

${ }^{a}$ Reaction conditions: DMM (7.5 mmol), Ru(acac) $)_{3}(1 \mathrm{~mol} \%)$, methanol (10 $\mathrm{mL}$ ), temp. $=393 \mathrm{~K}, p\left(\mathrm{H}_{2}\right)=7 \mathrm{MPa}$, time $=16 \mathrm{~h}^{b}{ }^{b}$ Mainly containing methyl acetate and methyl acrylate.

\section{2 反应条件的优化}

在确定配体结构和用量后, 我们对反应条件进行系 统的优化. 首先考察了温度和时间对 L1-Ru(acac) 3 体系 催化性能的影响, 结果如表 3 所示. 当温度为 $373 \mathrm{~K}$ 时, 反应体系活性较低, 难以有效催化 DMM 加氢(Entry 1, 表 3). 将反应温度提高到 $393 \mathrm{~K}$, 催化活性明显提高, 反 应 $8 \mathrm{~h}$ 即实现 44\% DMM 转化, 并取得 36\% 3-HPM 收率 (Entry 2, 表 3). 延长反应时间可进一步提高 DMM 转化 率和 3-HPM 收率(Entries $3 \& 4$, 表 3). 但是, 对比 Entry 3 和 Entry 4 数据可以看出, $393 \mathrm{~K}$ 反应 $16 \mathrm{~h}$ 后体系基本 达到平衡; 继续延长反应时间, 底物转化率和目标产物 收率无明显提高. 提高反应温度至 $413 \mathrm{~K}$, 虽然 $8 \mathrm{~h}$ 内体 系即实现 50\% DMM 转化, 但是目标产物 3-HPM 收率 较低, 仅为 $34 \%$ (Entry 5 , 表 3). 这可能是由于反应温度 过高导致发生 3-HPM $\beta$ 位羟基脱水等副反应所致. 因 此, $393 \mathrm{~K}$ 和 $16 \mathrm{~h}$ 是当前体系较为合适的反应温度和时 间.

在以上研究中, 采用甲醇作为溶剂. 在反应过程中, 甲醇一方面作为溶剂, 另一方面起到还原 $\mathrm{Ru}(\mathrm{acac})_{3}$ 生 成 $\mathrm{Ru}^{2+}, \mathrm{Ru}^{2+}$ 与配体配位原位生成催化剂的作用 ${ }^{[19]}$. 众 所周知, $\mathrm{Ru}$ 等过渡金属化合物与伯醇作用将诱导发生醇 脱羰基的反应 ${ }^{[20]}$. 在前期研究中, 我们发现羰基与 $\mathrm{Ru}$ 金属中心配位将占据催化剂活性位点, 导致催化剂中毒 和失活 ${ }^{[13 a]}$. 此外, Bergens 等 ${ }^{[21]}$ 报道指出小分子醇易与 $\mathrm{Ru}^{2+}$ 作用生成烷氧基配合物, 阻碍加氢过程的进行. 在 当前反应体系中, 过多甲醇的存在极有可能导致发生上
表 3 反应温度和时间对催化 DMM 加氢反应的影响 ${ }^{a}$ Table 3 Influence of reaction temperature and time on the catalytic hydrogenation of DMM

\begin{tabular}{cccccccc}
\hline \multirow{2}{*}{ Entry Temp./ } & Time/ & Conv./ & \multicolumn{4}{c}{ Yield/\% } \\
\cline { 5 - 8 } & $\mathrm{K}$ & $\mathrm{h}$ & $\%$ & 3-HPM & 1,3-PDO & MP & Others $^{b}$ \\
\hline 1 & 373 & 16 & 7 & 5 & 0 & 0 & 2 \\
2 & 393 & 8 & 44 & 36 & 0 & 2 & 6 \\
3 & 393 & 16 & 55 & 45 & 1 & 3 & 6 \\
4 & 393 & 24 & 58 & 47 & 2 & 4 & 5 \\
5 & 413 & 8 & 50 & 34 & 0 & 5 & 11 \\
\hline
\end{tabular}

${ }^{a}$ Reaction conditions: DMM (7.5 mmol), Ru(acac) $)_{3}(1 \mathrm{~mol} \%), \mathbf{L 1}(2 \mathrm{~mol} \%)$, methanol $(10 \mathrm{~mL}), p\left(\mathrm{H}_{2}\right)=7 \mathrm{MPa} .{ }^{b}$ Mainly containing methyl acetate and methyl acrylate.

述毒化过程. 因此, 我们采用甲醇和四氢呋喃(THF)混 合溶液作为溶剂, 并固定溶剂总体积为 $10 \mathrm{~mL}$, 考察了 甲醇用量对 $\mathbf{L 1}-\mathrm{Ru}(\mathrm{acac})_{3}$ 催化加氢性能的影响, 结果如 图 3 所示.

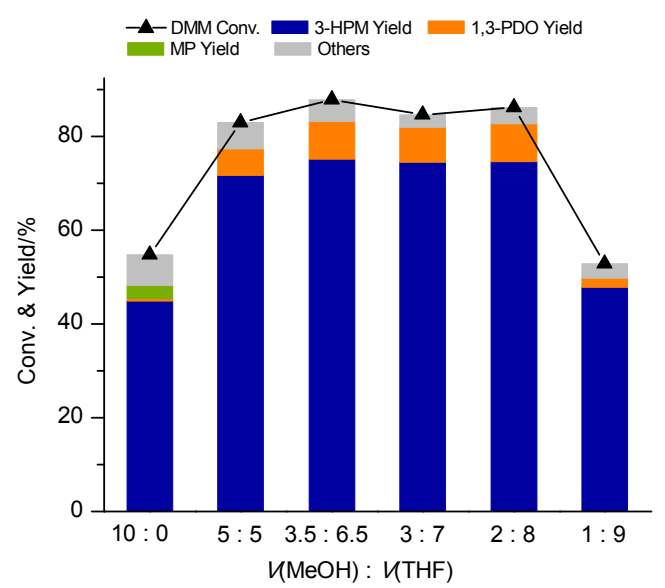

图 3 溶剂对 $\mathrm{L1}-\mathrm{Ru}(\mathrm{acac})_{3}$ 催化 DMM 加氢的影响

Figure 3 Influence of solvent on the catalytic hydrogenation of DMM

从图 3 结果可以看出, 相比于使用甲醇作为溶剂, DMM 转化率以及 3-HPM 收率随着反应体系中 THF 体 积的增加呈现出先增加后降低的近火山型变化趋势. 当 甲醇和 THF 体积分别为 2 和 $8 \mathrm{~mL}$ 时, 体系取得了较优 的催化反应结果，此时 DMM 转化率以及 3-HPM 收率分 别达到 $86 \%$ 和 $75 \%$, 同时还伴有 $8 \%$ 收率的二步加氢产 物 1,3-PDO 生成. 此外, 在甲醇和 THF 体积分别为 3 和 $7 \mathrm{~mL}$ (或 3.5 和 $6.5 \mathrm{~mL}$ 等)条件下, 也取得了优异的催化 反应结果. 以上结果说明, 在一定范围内采用甲醇-THF 混合溶剂可以有效提升 $\mathbf{L 1}-\mathrm{Ru}(\mathrm{acac})_{3}$ 催化体系的催化加 氢性能. 这主要得益于 THF 的引入, 有效减缓了反应体 系中甲醇与 $\mathrm{Ru}^{2+}$ 作用生成烷氧基配合物的可能, 以及 甲醇分解产生 $\mathrm{CO}$ 毒化催化剂的进程.

\section{3 底物的拓展}

考察了 L1-Ru(acac) 3 催化不同结构酯类分子加氢的 反应性能, 结果如表 4 所示. 表 4 中所用到酯类分子结 
构见图 2. 从表 4 结果可以看出, $\mathrm{L1}-\mathrm{Ru}(\mathrm{acac})_{3}$ 难以有效 催化 3-HPM 加氢制 1,3-PDO. 这一结果与 $\mathbf{L 1}-\mathrm{Ru}(\mathrm{acac})_{3}$ 催化 DMM 加氢取得 8\% 1,3-PDO 收率的结果一致(Entry 1, 表 4), 导致出现这一现象的原因还有待进一步探究. 相比于催化稳定性较差的 3-HPM 加氢, L1-Ru(acac) $)_{3}$ 在 较高温度下可以有效催化 2-羟基丙酸甲酯(E3)加氢制 1,2-丙二醇(Entry 3, 表 4). L1-Ru(acac) 3 在较低温度下也 可以有效催化草酸酯分子(E4 \& E5)部分加氢制得乙醇 酸酯(Entries 4 \& 5, 表 4). 进一步研究发现, L1-Ru$(\mathrm{acac})_{3}$ 催化 $\gamma$-戊内酯(E6)加氢制 1,4-戊二醇, 可以取得 近乎完全的底物转化率和目标产物收率(Entry 6, 表 4).

表 4 其它酯类化合物的催化加氢反应 ${ }^{a}$

Table 4 Substrate scope for the catalytic system

\begin{tabular}{|c|c|c|c|c|c|}
\hline \multirow{2}{*}{ Entry } & \multirow{2}{*}{ Substrate } & \multirow{2}{*}{ Temp./K } & \multirow{2}{*}{ Conv. $/ \%$} & \multicolumn{2}{|c|}{ Hydrogenation yield/\% } \\
\hline & & & & Product $\mathrm{A}^{b}$ & Product $\mathrm{B}^{c}$ \\
\hline 1 & E1 & 393 & 86 & 75 (3-HPM) & $8(1,3-P D O)$ \\
\hline 2 & E2 & 393 & 7 & 3 (1,3-PDO) & - \\
\hline 3 & $\mathbf{E 3}$ & 423 & 83 & 82 (1,2-PDO) & - \\
\hline 4 & E4 & 388 & 100 & 98 (MG) & $1(\mathrm{EG})$ \\
\hline $5^{d}$ & E5 & 383 & 98 & 97 (MPEG) & $1(\mathrm{EG})$ \\
\hline 6 & E6 & 413 & 97 & 97 (1,4-PDO) & - \\
\hline $7^{d}$ & E7 & 413 & 63 & $59(\mathrm{EG})$ & $54(\mathrm{MeOH})$ \\
\hline
\end{tabular}

${ }^{a}$ Reaction conditions: Substrate $(7.5 \mathrm{mmol}), \mathrm{Ru}(\mathrm{acac})_{3}(1 \mathrm{~mol} \%), \mathbf{L 1}$ (2 $\mathrm{mol} \%$ ), $2 \mathrm{~mL}$ methanol and $8 \mathrm{~mL}$ THF as the solvent, $P_{(\mathrm{H} 2)}=7 \mathrm{MPa}$, Time $=16$ h. ${ }^{b}$ Yield of product A. ${ }^{c}$ Yield of product B. ${ }^{d} 2 \mathrm{~mL}$ ethanol and $8 \mathrm{~mL}$ THF as the solvent. MG: methyl glycolate; EG: ethylene glycol; MPEG: ethyl glycolate; 1,2-PDO: 1,2-propanediol; 1,4-PDO: 1,4-pentanediol.
值得注意的是, $\mathbf{L 1}-\mathrm{Ru}(\mathrm{acac})_{3}$ 在催化碳酸乙烯酯(E7)加 氢制甲醇和乙二醇的反应中, 也表现出了一定的催化活 性(Entry 7, 表 4). 作为碳酸酯的一种, 碳酸乙烯酯可以 从环氧乙烷和 $\mathrm{CO}_{2}$ 制备得到 ${ }^{[22]}$. 然而, 由于共轭结构的 存在, 碳酸酯稳定性很高, 较难被加氢 ${ }^{[23]}$. Ding 等 ${ }^{[11]}$ 报 道指出，耦合碳酸乙烯酯制备及其加氢的“omega过程”, 在催化 $\mathrm{CO}_{2}$ 和环氧乙烷转化制甲醇和乙二醇之间架起 了一座新的桥梁. 因此, Ding 等认为高效催化碳酸乙烯 酯加氢制甲醇是解决全球能源问题的理想方案之一.

\section{4 推测的反应机理}

基于文献报道以及我们课题组前期的工作基 础 ${ }^{[11,13 a, 13 b, 24]}$, 推测了如图 4 所示的 $\mathbf{L 1}-\mathrm{Ru}(\mathrm{acac})_{3}$ 催化 DMM 加氢的反应机理. 首先, 在原位条件下反应形成 同时具有 $\mathrm{Ru}-\mathrm{H}$ 和 $\mathrm{N}-\mathrm{H}$ 官能团的催化活性物种 a. a 通过 $\mathrm{Ru}-\mathrm{H}$ 与 $\mathrm{N}-\mathrm{H}$ 分别与 $\mathrm{DMM}$ 羰基作用形成过渡 态 TS1，进一步转化生成 3-氧代丙酸甲酯和甲醇以及催 化剂中间体 b. 中间体 $\mathbf{b}$ 可以通过活化氢气分子返回到 活性态 $\mathbf{a}$, 实现催化循环. 最后, 中间产物 3-氧代丙酸 甲酯与 $\mathbf{a}$ 通过形成过渡态 $\mathbf{T S 2}$, 最终转化生成目标产物 3-HPM. 综合以上反应过程可以看出，a 是通过结构中 $\mathrm{N}-\mathrm{H}$ 和 $\mathrm{Ru}-\mathrm{H}$ 的协同作用实现加氢反应的催化循环. 由不含 $\mathrm{N}-\mathrm{H}$ 官能团的 $\mathbf{L 2}$ 配体与 $\mathrm{Ru}(\mathrm{acac})_{3}$ 所构成的催 化体系, 在反应条件下没有催化活性的实验结果验证了 这一反应机理(Entry 2, 表 1).

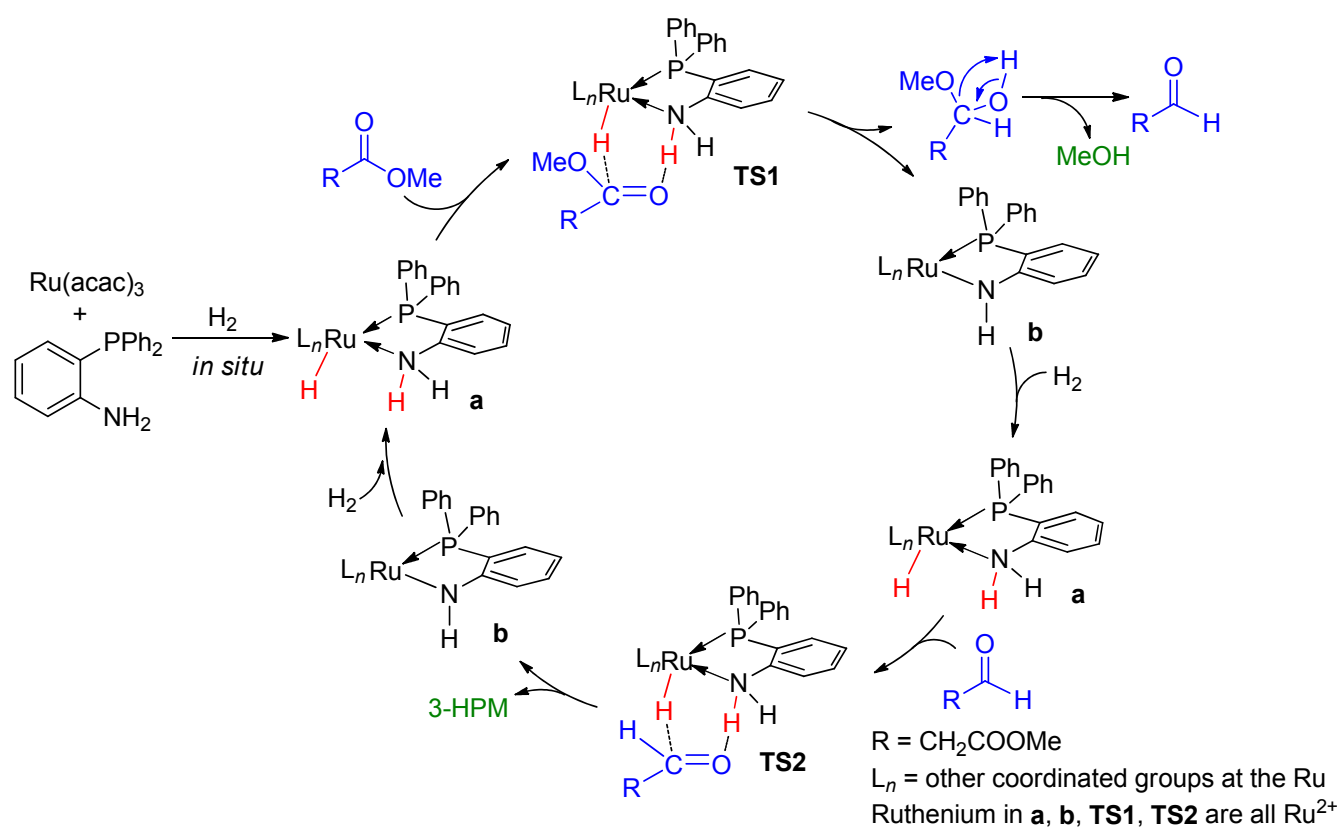

图 4 DMM 催化加氢制 3-HPM 可能的反应机理图

Figure 4 Proposed reaction mechanism for the reduction of DMM into 3-HPM 


\section{2 结论}

首次以 $\mathrm{Ru}(\mathrm{acac})_{3}$ 和 $o$-二苯基膦苯胺构成均相催化 剂体系, 应用于 DMM 加氢制 3-HPM 或 1,3-PDO, 系统 探究了配体用量、反应温度和时间以及溶剂等反应条件 对催化性能的影响. 研究发现, $\mathrm{Ru}(\mathrm{acac})_{3}$ 和 $o$-二苯基膦 苯胺物质的量比为 $1: 2$ 较为合适; 此外, 相比于使用甲 醇作为溶剂, 采用甲醇-THF 混合溶剂可有效提升催化 体系的催化加氢性能. 在优化的反应条件下, DMM 转 化率达到 86\%, 目标产物 3-HPM 和 1,3-PDO 收率分别 为 $75 \%$ 和 $8 \%$. 这一催化体系在一定反应条件下也可以 有效催化草酸酯、 $\gamma$-戊内酯、碳酸乙烯酯等多种酯类分 子的加氢.

\section{3 实验部分}

\section{1 仪器与试剂}

催化剂活性评价采用配备有聚四氟乙烯内祄的 PARR 5500 型高压反应釜，釜体积为 $100 \mathrm{~mL}$. 反应液采 用配备有氢焰离子化检测器(FID)、KB-Wax 色谱柱(60 $\mathrm{m} \times 0.32 \mathrm{~mm} \times 0.33 \mu \mathrm{m}$ ) 以及香港 Collect 公司生产的自 动进样器(AS-2920)的福立-9790II 型气相色谱进行分析.

甲苯、己烷、THF 以及甲醇等有机溶剂购买自国药 集团上海试剂公司; $\mathrm{Ru}(\mathrm{acac})_{3}$ 、配体合成所需原料、 DMM 等酯类化合物以及相应的加氢产物购买自 Aldrich、Alfa Aesar、百灵威以及阿拉丁等化学试剂公 司. 文中所使用配体 $\mathbf{L 1} \sim \mathbf{L 8}$ 参考文献方法合成 ${ }^{[25]}$.

\section{2 实验方法}

实验中涉及无氧无水的操作均采用标准 Schlenk 技 术或在 Mbraum 手套箱 $\left(\mathrm{O}_{2}\right.$ 和 $\mathrm{H}_{2} \mathrm{O}$ 含量低于 $\left.1.0 \mathrm{ppm}\right)$ 中 进行. 有机溶剂甲苯、已烷、THF 等用钠丝预处理后, 氮 气气氛下用钠钾合金回流后取用. DMM 等液体酯类用 氢化钻或无水硫酸镁室温摚拌 $2 \mathrm{~d}$ 后氮气气氛下蒸馏, 储存在手套箱中备用. 草酸二甲酯和碳酸乙烯酯直接减 压蒸馏后储存在手套箱中备用.

以催化 DMM 加氢为例说明活性评价过程: 首先, 称取一定量 $\mathrm{Ru}(\mathrm{acac})_{3}$ 、配体、 $\mathrm{DMM}$ 以及溶剂于釜体中, 反应釜组装完成后转移出手套箱; 紧接着, 用冰水冷却 釜体至约 $278 \mathrm{~K}$, 用 $\mathrm{H}_{2}$ 洗气三次并充 $\mathrm{H}_{2}$ 压力至 $7 \mathrm{MPa}$. 然后, 将釜体置于加热装置中加热升温至指定温度并维 持一定时间; 反应完成后, 快速将釜体冷却至约 $278 \mathrm{~K}$ 并排去釜中残余的氢气. 最后, 在反应液中加入一定量 对二甲苯作为内标, 搅拌均匀后用气相色谱进行分析. 气相色谱分析具体参数如下: 采用 $\mathrm{N}_{2}$ 作为载气, 并固定 其流速为 $25 \mathrm{~mL} / \mathrm{min}$; 气化室和 FID 检测器温度分别为 533 和 $523 \mathrm{~K}$; 程序升温过程如下: $313 \mathrm{~K}$ 维持 $5 \mathrm{~min}$, 后
以 $10 \mathrm{~K} / \mathrm{min}$ 升高温度至 $473 \mathrm{~K}$ 并在这一温度下维持 20 min. 根据所得谱图中产物、原料的峰面积进行转化率和 产率的计算.

辅助材料(Supporting Information) 部分活性数据及 反应产物的气相色谱图. 这些材料可以免费从本刊网站 (http://sioc-journal.cn/)上下载.

\section{References}

[1] Kraus, G. A. Clean 2008, 36, 648.

[2] Arntz, D.; Wiegand, N. US 5015789, 1991.

[3] (a) Slaugh, L. H.; Weider, P. R. US 5256827, 1993.

(b) Powell, J. B.; Mullin, S. B.; Weider, P. R.; Eubanks, D. C.; Arhancet, J. P. US 5770776, 1998.

[4] (a) Kaur, G.; Srivastava, A. K.; Chand, S. Biochem. Eng. J. 2012, $64,106$.

(b) Lee, C. S.; Aroua, M. K.; Daud, W. M. A. W.; Cognet, P.; Pérès-Lucchese, Y.; Fabre, P. L.; Reynes, O.; Latapie, L. Renewable Sustainable Energy Rev. 2015, 42, 963.

[5] (a) Wang, Y.; Zhou, J.; Guo, X. RSC Adv. 2015, 5, 74611. (b) Sun, D.; Yamada, Y.; Sato, S.; Ueda, W. Appl. Catal. B Environ. 2016, 193, 75 .

[6] (a) Chen, L. F.; Guo, P. J.; Qiao, M. H.; Yan, S. R.; Li, H. X.; Shen, W.; Xu, H. L.; Fan, K. N. J. Catal. 2008, 257, 172.

(b) He, Z.; Lin, H.; He, P.; Yuan, Y. J. Catal. 2011, 277, 54.

(c) Peng, S. Y.; Xu, Z. N.; Chen, Q. S.; Chen, Y. M.; Sun, J.; Wang, Z. Q.; Wang, M. S.; Guo, G. C. Chem. Commun. 2013, 49, 5718.

(d) Ma, X. B.; Chi, H. W.; Yue, H. R.; Zhao, Y. J.; Xu, Y.; Lv, J.; Wang, S. P.; Gong, J. L. AIChE J. 2013, 59, 2530.

[7] (a) Ding, T.; Tian, H.; Liu, J.; Wu, W.; Zhao, B. Catal. Commun. 2016, 74,10

(b) Ding, T.; Tian, H.; Liu, J.; Wu, W.; Yu, J. Chin. J. Catal. 2016, $37,484$.

[8] (a) He, L.; Gong, X.; Ye, L.; Duan, X.; Yuan, Y. J. Energy Chem. 2016, 25, 1038.

(b) Yu, J.; Cao, J.; Du, L.; Wei, Y.; Wang, T.; Tian, H. Appl. Catal., A 2018, 555, 161.

[9] (a) Zhao, B. G.; Han, Z. B.; Ding, K. L. Angew. Chem., Int. Ed. 2013, 52, 4744.

(b) Werkmeister, S.; Junge, K.; Beller, M. Org. Process Res. Dev. 2014, 18, 289.

(c) Pritchard, J.; Filonenko, G. A.; van Putten, R.; Hensen, E. J. M.; Pidko, E. A. Chem. Soc. Rev. 2015, 44, 3808.

[10] Li, W.; Xie, J. H.; Yuan, M. L.; Zhou, Q. L. Green Chem. 2014, 16, 4081.

[11] Han, Z.; Rong, L.; Wu, J.; Zhang, L.; Wang, Z.; Ding, K. Angew. Chem., Int. Ed. 2012, 51, 13041

[12] (a) Tan, X.; Wang, Y.; Liu, Y.; Wang, F.; Shi, L.; Lee, K. H.; Lin, Z.; Lv, H.; Zhang, X. Org. Lett. 2015, 17, 454.

(b) Tan, X.; Wang, Q.; Liu, Y.; Wang, F.; Lv, H.; Zhang, X. Chem. Commun. 2015, 51, 12193.

[13] (a) Fang, X.; Zhang, C.; Chen, J.; Zhu, H.; Yuan, Y. RSC Adv. 2016, 6, 45512 .

(b) Fang, X.; Sun, M.; Zheng, J.; Li, B.; Ye, L.; Wang, X.; Cao, Z.; Zhu, H.; Yuan, Y. Sci. Rep. 2017, 7, 3961.

(c) Zhang, Y. W.; Chen, Y. L.; Fang, X. L.; Yuan, Y. Z.; Zhu, H. P. Chin. J. Org. Chem. 2017, 37, 2275 (in Chinese).

(张亦伟，陈艺林，方霄龙，袁友珠，朱红平，有机化学，2017， 37, 2275.)

[14] Ohkuma, T.; Ooka, H.; Hashiguchi, S.; Ikariya, T.; Noyori, R. J. Am. Chem. Soc. 1995, 117, 2675.

[15] Teunissen, H. T.; Elsevier, C. J. Chem. Commun. 1997, 667.

[16] Teunissen, H. T.; Elsevier, C. J. Chem. Commun. 1998, 1367.

[17] (a) Geilen, F. M. A.; Engendahl, B.; Harwardt, A.; Marquardt, W.; 
Klankermayer, J.; Leitner, W. Angew. Chem., Int. Ed. 2010, 49, 5510 .

(b) Wesselbaum, S.; vom Stein, T.; Klankermayer, J.; Leitner, W. Angew. Chem., Int. Ed. 2012, 51, 7499.

[18] Saudan, L. A.; Saudan, C. M.; Debieux, C.; Wyss, P. Angew. Chem., Int. Ed. 2007, 46, 7473 .

[19] Geilen, F. M. A.; Engendahl, B.; Hölscher, M.; Klankermayer, J.; Leitner, W. J. Am. Chem. Soc. 2011, 133, 14349.

[20] (a) Van der Sluys, L. S.; Kubas, G. J.; Caulton, K. G. Organometallics 1991, 10, 1033.

(b) Chen, Y. Z.; Chan, W. C.; Lau, C. P.; Chu, H. S.; Lee, H. L.; Jia, G. Organometallics 1997, 16, 1241.

[21] Hamilton, R. J.; Bergens, S. H. J. Am. Chem. Soc. 2006, 128, 13700.

[22] Mirza, C.; Christian, B.; Bernhard, R.; Wolfgang, A. H.; Fritz, E. K. Angew. Chem., Int. Ed. 2011, 50, 8510.

[23] Ito, M.; Ootsuka, T.; Watari, R.; Shiibashi, A.; Himizu, A.; Ikariya, T. J. Am. Chem. Soc. 2011, 133, 4240 .
[24] (a) John, J. M.; Takebayashi, S.; Dabral, N.; Miskolzie, M.; Bergens, S. H. J. Am. Chem. Soc. 2013, 135, 8578.

(b) Tan, X.; Wang, Y.; Liu, Y.; Wang, F.; Shi, L.; Lee, K. H.; Lin, Z.; Lv, H.; Zhang, X. Org. Lett. 2015, 17, 454.

[25] (a) Herd, O.; Heßler, A.; Hingst, M.; Tepper, M.; Stelzer, O. J. Organomet. Chem. 1996, 522, 69.

(b) Hingst, M.; Tepper, M.; Stelzer, O. Eur. J. Inorg. Chem. 1998 1998, 73.

(c) Habtemariam, A.; Watchman, B.; Potter, B. S.; Palmer, R.; Parsons, S.; Parkin, A.; Sadler, P. J. J. Chem. Soc., Dalton Trans. 2001 1306.

(d) Doherty, S.; Knight, J. G.; Scanlan, T. H.; Elsegood, M. R. J.; Clegg, W. J. Organomet. Chem. 2002, 650, 231.

(e) Han, F. B.; Zhang, Y. L.; Sun, X. L.; Li, B. G.; Guo, Y. H.; Tang, Y. Organometallics 2008, 27, 1924.

(f) Richard, V.; Ipouck, M.; Mérel, D. S.; Gaillard, S.; Whitby, R. J.; Witulski, B.; Renaud, J. L. Chem. Commun. 2014, 50, 593.

(Zhao, X.) 